\title{
Estado da arte em revistas educacionais sobre estudos feitos com famílias de crianças com necessidades educacionais especiáis no período de 2002 a 2011
}

Giovana Mendes Ferroni*

Fabiana Cia**

\section{Resumo}

O objetivo deste artigo é mapear os estudos indexados entre os anos 2002 e 2011 na área da educação, e avaliados como Al e A2 pelo banco de dados Qualis Capes, no triênio 2010-2012, a fim de verificar de que maneiras as pesquisas com famílias de crianças com necessidades educacionais especiais têm sido feitas. Para isso, foram analisados os seguintes elementos: evolução anual dos estudos, sujeitos analisados, temáticas adotadas e instrumentos de coleta de dados utilizados, a partir de buscas na base de dados Scielo e Periódicos Capes e utilizadas uma multiplicidade de descritores combinados entre si. O critério estabelecido para considerar família foi o de que as pesquisas deveriam conter as palavras-chaves: família, relações familiares, relações parentais, pais e irmãos intercruzadas com: educação especial, pessoas com necessidades educacionais especiais, educação inclusiva, inclusão, autismo, autista, superdotação, altas habilidades, paralisia cerebral, baixa visão, cegueira, surdez, transtorno global do desenvolvimento, síndrome de Down, deficiência, deficiência intelectual, deficiência física, deficiência visual, surdez e deficiência múltipla. Portanto, os artigos foram obtidos pelo entrecruzamento desses descritores, mas selecionados aqueles que continham uma dessas palavras. Dessa forma, foi encontrado um total de 36 artigos nacionais indexados em revistas da área da Educação. Os dados mostraram que houve um crescimento de pesquisas empíricas nessa área.

Palavras-chave: Educação Especial; Família; Necessidades educacionais especiais.

\footnotetext{
* Doutoranda em Educação Especial da Universidade Federal de São Carlos, São Carlos, São Paulo, Brasil.

** Professora Doutora do Departamento de Psicologia e do Programa de Pós-Graduação em Educação Especial. Universidade Federal de São Carlos, São Carlos, São Paulo, Brasil. Apoio financeiro Fapesp/FMCSV, CNPq.
} 


\section{State of the art in educational journals on studies of families of children with special educational needs in the period from 2002 to 2011}

\section{Abstract}

The purpose of this paper is mapping studies indexed between the years of 2002 and 2011 in the educational field and evaluated like $\mathrm{Al}$ or $\mathrm{A} 2$ by the database Qualis Capes, in the three years 2010-2012, in order to research ways that studies with families of children with special educational needs have been made. For this, we analyze elements such as: annual evolution of the studies, subjects analyzed, adopted and thematic instruments used to collect data from database searches "Scielo" and "Capes Journal" and a multitude of descriptors combined. The criterion for considering the family was that the research should contain the following keywords: family, family relationships, parental relationships, parents and siblings crisscrossing: special education, people with special educational needs, inclusive education, inclusion, autism, autistic, gifted, high ability, cerebral palsy, low vision, blindness, deafness, pervasive developmental disorder, Down Syndrome, disability, intellectual disability, physical disability, visual impairment, hearing impairment and multiple disabilities. Thus, the papers were obtained by intercrossing these descriptors, but it was selected those containing one of these words. With this, we found a total of 36 papers in national journals indexed in the area of Education. The data showed that there was a growing body of empirical research in this area.

Keywords: Special Education; Family; Special educational needs.

\section{Introdução}

A família é o primeiro contexto de socialização da criança e desempenha papel fundamental em seus desenvolvimentos físico, emocional e cognitivo. Dessa forma, conceber a família como promotora do desenvolvimento exige compreendê-la como um sistema de interações e relações que se influencia reciprocamente, isto é, qualquer mudança que ocorre nesse sistema afeta a todos (DESSEN, 1997; DESSEN; BRAZ, 2005; DESSEN; CERQUEIRA-SILVA, 2008; JUNIOR; MESSA, 2007; PANIAGUA, 2004). De acordo com Dessen (1997), Dessen e Braz (2005), nos últimos anos, houve um crescimento do número de pesquisas que retratam a influência da família no desenvolvimento da criança. Essas pesquisas trazem dados importantes, pois não focam somente as interações entre as díades, como, por exemplo, mãe-criança e paicriança, mas se interessaram também pelas relações que são estabelecidas entre todas as pessoas que compõem o grupo familiar.

O conceito de interação e relação que as autoras acima se referem é o mesmo compreendido por Dessen e Lewis (1998), e está baseado nas conceituações de 
Hinde $(1979,1997)$ que define interação como contextos em que há, no mínimo, duas pessoas, sendo que o conceito de relação parte do mesmo princípio da interação, mas envolve contextos mais amplos, que são influenciados pelas interações anteriores do passado, do presente e do futuro. Assim,

estudar o desenvolvimento familiar envolve, necessariamente, estudar os processos de comunicação e as interações e relações existentes entre os membros da família, levando em consideração a fundamental importância do contexto sócio-históricocultural. (DESSEN; BRAZ, 2005, p. 128)

Quando se trata de crianças com necessidades educacionais especiais (NEE), as interações e as relações são bastante afetadas (DESSEN; CERQUEIRASILVA, 2008; PANIAGUA, 2004; SILVA; DESSEN, 2001), isso porque os pais vivenciam diversas reações até se adaptarem à deficiência do filho; entre elas: (a) fase de choque, na qual algumas famílias assustam ou ficam paralisadas emocionalmente ao receberem a notícia de que o filho possui algum tipo de deficiência; (b) fase de negação, na qual as famílias negam ou não acreditam que o filho possui deficiência; (c) fase de reação, a qual pode ser expressa na forma de irritação, culpa e depressão; (d) fase de adaptação e de orientação, na qual a família já compreende a deficiência do filho e procura ajuda.

Geralmente, essas fases não são superadas rapidamente pelas famílias e, algumas delas, podem se repetir. No entanto, uma parte dos pais consegue se adaptar e superar tais dificuldades, sendo que alguns afirmam se alegrar com cada conquista do filho; eles valorizam os esforços, admiram e se orgulham das superações dos filhos, embora façam isso expressando um pouco de preocupação (PANIAGUA, 2004).

Apesar de a maioria das famílias se adaptarem às novas demandas, pais também vivenciam muitas situaçoes que podem gerar estresse, como, por exemplo: (a) passagem por diferentes tratamentos e profissionais; (b) escolha sobre o tipo de educação que será oferecida ao filho; (c) aumento da dedicação dos pais aos filhos acometidos por alguma deficiência, pois, a depender do tipo de NEE, a criança requer muitos cuidados físicos e mais estimulações; (d) dificuldade em encontrar pessoas que cuidem da criança na ausência dos pais, fazendo com que um dos cônjuges se sacrifique para ficar com o filho, o que, na maioria das vezes, é atribuído à mãe; (e) aumento dos gastos econômicos e, (f) preocupação com o futuro do filho (PANIAGUA, 2004).

A adaptação dos pais à criança com NEE torna-se mais importante, considerando que a maneira como eles lidam com a deficiência do filho influenciará o próprio filho a lidar com a sua deficiência (SILVA; DESSEN, 2001). Nesse sentido, Castro e Piccinini, (2002) afirmam que a família deve atenuar os efeitos negativos na sua maneira de lidar com a situação a fim de criar um ambiente estimulador e favorável ao desenvolvimento da criança. 
Dessen e Cerqueira-Silva (2008) apontam para a importância de se criar Programas de Educação Familiar (PEF) que deem suportes às famílias de crianças com NEE, a fim torná-las facilitadoras e promotoras do desenvolvimento de seus filhos. Esses programas têm como finalidade um processo educativo que consiste em treinar, orientar, ensinar, informar toda a família (mãe, pai e filhos), objetivando torná-los participantes da educação do filho com NEE. Entretanto, é preciso respeitar e reconhecer os conhecimentos que a família possui do filho, pois, dessa forma, ela se sentirá reconhecida e terá estímulo para procurar as alternativas melhores para a educação e o desenvolvimento da criança com NEE. Assim, a parceria com a família envolve empoderá-la, ou seja, "passa de uma posição passiva (de ser mera receptora de serviços) para ser um agente de transformação social, capaz de mudar e enfrentar com dignidade as múltiplas adversidades da vida" (WILLIAMS; AIELLO, 2004). Para embasar tais programas, deve-se conhecer as principais características das famílias de crianças com NEE.

Assim, entende-se que, quando se trata de família de crianças com NEE como promotoras do seu desenvolvimento, ressalta-se a importância de compreender todo o contexto da criança, e como as relações são estabelecidas entre ela e todos os membros. Portanto, o objetivo desse artigo é mapear os estudos indexados entre os anos de 2002 e 201l, na área da educação, e avaliados pelo banco de dados Qualis Capes como Al e A2, no triênio 2010-2012, a fim de verificar como têm sido feitas as pesquisas com famílias de crianças com NEE, a partir da análise dos seguintes elementos: evolução anual dos estudos, sujeitos analisados, temáticas adotadas e instrumentos de coleta de dados utilizados. Dessa forma, a pesquisa poderá contribuir para o campo acadêmico no sentido de elaborar um mapeamento dos principais estudos da área, para, com isso, identificar os elementos mais e menos recorrentes nas pesquisas, ajudando o desenvolvimento de novos estudos que tratem da família de crianças com NEE.

\section{Método}

Foi realizada uma busca na base de dados Scielo e Periódicos Capes, indexados na área da educação, no período compreendido entre 2002 e 201l, e avaliados pelo banco de dados Qualis Capes como Al e A2, no triênio 2010-2012. Para isso, foram utilizados múltiplos descritores, combinados entre si, de modo a abarcar a maior quantidade de periódicos que continham a temática relacionada à família de pessoas com NEE. O critério estabelecido para considerar família foi o de que as pesquisas deveriam conter as palavras-chaves: família, relações familiares, relações parentais, pais, irmãos entrecruzadas com as: educação especial, pessoas com necessidades educacionais especiais, educação inclusiva, inclusão, autismo, autista, superdotação, altas habilidades, paralisia cerebral, baixa visão, cegueira, surdez, transtorno global do desenvolvimento, síndrome de Down, deficiência, deficiência intelectual, deficiência física, deficiência visual, surdez e deficiência múltipla. Assim, os artigos foram obtidos pelo entrecruzamento desses descritores e selecionados os que continham uma dessas palavras. Desta forma, foi encontrado um total de 36 artigos nacionais indexados em revistas da área da Educação. 
Para a análise dos dados, foram criadas tabelas cujos conteúdos abarcavam: público-alvo, locais onde foram publicados os artigos (nome das revistas), periodicidade anual das publicações (quantidade de artigos publicados em cada período), técnica de coleta de dados utilizadas nas pesquisas e as temáticas investigadas nas publicações. Tais temáticas foram criadas a partir da leitura dos objetivos das pesquisas e sua posterior categorização. Depois disso, procurou dialogar os resultados encontrados com a produção acadêmica da área. Ressalta-se que, em todas as tabelas, serão encontrados o valor $\mathrm{N}$, que se refere à quantidade de artigos publicados, e \% que se refere aos valores das porcentagens aproximadas encontradas em todos os dados das tabelas.

\section{Resultados}

Consta na Tabela l a categorização do público-alvo dos artigos selecionados.

\begin{tabular}{|l|c|c|}
\hline \multicolumn{1}{|c|}{ Público-alvo } & N & $\%$ \\
\hline Síndrome de Down & 8 & 22,22 \\
\hline Diferentes Populações & 5 & 13,88 \\
\hline Surdez & 5 & 13,88 \\
\hline Autismo & 3 & 8,33 \\
\hline Altas habilidades & 2 & 5,55 \\
\hline Deficiência intelectual & 2 & 5,55 \\
\hline Deficiência multipla & 2 & 5,55 \\
\hline Alterações de linguagem de origem neurológica & 1 & 2,77 \\
\hline Deficiência física & 1 & 2,77 \\
\hline Deficiência visual & 1 & 2,77 \\
\hline Distúrbio de linguagem & 1 & 2,77 \\
\hline Paralisia cerebral & 1 & 2,77 \\
\hline Transtorno de déficit de atenção e hiperatividade & 1 & 2,77 \\
\hline Transtornos globais do desenvolvimento & 1 & 2,77 \\
\hline Criança de risco & 1 & 2,77 \\
\hline Criança com desenvolvimento típico & 1 & 2,77 \\
\hline TOTAL & 36 & $100 \%$ \\
\hline
\end{tabular}

Tabela 1. Categorização do público-alvo

Segundo dados da Tabela l, observa-se que as populações mais estudadas foram síndrome de Down (CAMARGO; TOREZAN, 2004; DALLABRIDA, 2007; HENN; PICCININI; GARCIAS, 2008; LUIZ et al., 2008; PETEAN; SUGUIHURA, 2005; PEREIRA-SILVA; DESSEN, 2007; SILVA; DESSEN, 2003; SILVA; DESSEN, 2006), totalizando oito publicações, o que corresponde a, aproximadamente, 22,22\% do montante de artigos encontrados e, em seguida, os estudos feitos com diferentes populações (BARBOSA; ROSINI; PEREIRA, 2007; BOLSONI-SILVA et al., 2010; 
FERRAZ; ARAÚJO; CARREIRO, 2010; SANINI et al., 2008; SILVA; MENDES, 2008) e surdez (CANHO; NEME; YAMADA, 2006; MOTTI; PARDO, 2010; SCHEMBERG; GUARINELLO; SANTANA, 2009; SILVA; PEREIRA; ZANOLLI, 2007; YAMANAKA et al., 2010), ambas totalizando cinco publicações, o que equivale a 13,88\%. É importante ressaltar que o termo diferentes populações refere-se à estudos realizados com públicos diversificados que incluíam tanto pessoas com NEE, quanto pessoas com desenvolvimento típico e atraso no desenvolvimento. A Tabela 2 apresenta a porcentagem de artigos que cada revista publicou no período de 2002 a 2011.

\begin{tabular}{|c|c|c|c|c|c|}
\hline Periódicos & $\mathrm{N}$ & $\%$ & Qualis & $\mathrm{N}$ & $\%$ \\
\hline Psicologia: Reflexão e Crítica & 6 & 16,66 & $\mathrm{Al}$ & 6 & 16,66 \\
\hline Revista Brasileira de Educação Especial & 20 & 55,55 & A2 & \multirow{5}{*}{30} & \multirow{5}{*}{83,33} \\
\hline Psicologia: Teoria e Pesquisa & 5 & 13,88 & A2 & & \\
\hline Psicologia em Estudo & 2 & 5,55 & A2 & & \\
\hline Estudos de Psicologia (Campinas) & 2 & 5,55 & A2 & & \\
\hline Estudos de Psicologia (Natal) & 1 & 2,77 & A2 & & \\
\hline TOTAL & 36 & $100 \%$ & & & \\
\hline
\end{tabular}

Tabela 2. Porcentagem de artigos por periódicos

O periódico com maior publicação foi a Revista Brasileira de Educação Especial (BARBOSA; ROSINI; PEREIRA, 2007; BOLSONI-SILVA et al., 2010; CAMARGO; TOREZAN, 2004; CIA; WILLIAMS; AIELLO, 2005; CHACON, 2011; CHAGAS; FLEITH, 2009; DALLABRIDA, 2007; DELIBERATO; MANZINI; GUARDA, 2004; FERRAZ; ARAÚJO; CARREIRO, 2010; GRAÇA et al., 2010; KRÜGER et al., 2011; LUIZ et al., 2008; MOTTI; PARDO, 2010; PEREIRA-SILVA; DESSEN, 2007; PETEAN; SUGUIHURA, 2005; POSTALLI; MALASPINA; LAMÔNICA, 2004; MUNUERA; AIELLO, 2011; SCHEMBERG; GUARINELLO; SANTANA, 2009; SILVA; MENDES, 2008; TAKASE; CHUN, 2010), totalizando 20 publicações e uma porcentagem de 55,55\%. O segundo periódico de maior publicação foi o de Psicologia: Reflexão e Crítica (BELLÉ et al., 2009; FÁVERO; SANTOS, 2005; NUNES; AIELLO, 2008; SANINI et al., 2008; SILVA; DESSEN, 2003; SILVA; DESSEN, 2006), com um total de seis artigos. O terceiro periódico com maior publicação foi o de Psicologia: Teoria e Pesquisa (CHAGAS; FLEITH, 2011; SILVA; PEREIRA; ZANOLLI, 2007; SILVEIRA; NENES, 2006; SOARES; FRANCO; CARVALHO, 2009; YAMANAKA et al., 2010) totalizando cinco publicações, o que equivale a 13,88\%.

Ainda, na tabela 2, observa-se, por um lado, que os periódicos com Qualis A2, entre eles: Revista Brasileira de Educação Especial (BARBOSA; ROSINI; PEREIRA, 2007; BOLSONI-SILVA et al., 2010; CAMARGO; TOREZAN, 2004; CIA; WILLIAMS; AIELLO, 2005; CHACON, 2011; CHAGAS; FLEITH, 2009; DALLABRIDA, 2007; DELIBERATO; MANZINI; GUARDA, 2004; FERRAZ; ARAÚJO; CARREIRO, 2010; GRAÇA et al., 2010; KRÜGER et al., 2011; LUIZ et al., 2008; MALASPINA; LAMÔNICA, 2004; MOTTI; PARDO, 2010; PEREIRA-SILVA; DESSEN, 2007; PETEAN; SUGUIHURA, 2005; POSTALLI; MUNUERA; AIELLO, 2011; 
SCHEMBERG; GUARINELLO; SANTANA, 2009; SILVA; MENDES, 2008; TAKASE; CHUN, 2010); Psicologia: Teoria e Pesquisa (CHAGAS; FLEITH, 2011; SILVA; PEREIRA; ZANOLLI, 2007; SILVEIRA; NENES, 2006; SOARES; FRANCO; CARVALHO, 2009; YAMANAKA et al., 2010); Psicologia em Estudo (HENN; PICCININI; GARCIAS, 2008; SIFUENTES; BOSA, 2010); Estudos de Psicologia (Campinas) (CANHO; NEME; YAMADA, 2006; KREUTZ; BOSA, 2009); Estudos de Psicologia (Natal) (GOMES; BOSA, 2004) tiveram a maior incidência de publicação, totalizando 30 publicações, o que corresponde a 83,33\%. Por outro lado, o periódico Psicologia: Reflexão e Crítica (BELLÉ et al., 2009; FÁVERO; SANTOS, 2005; NUNES; AIELLO, 2008; SANINI et al., 2008; SILVA; DESSEN, 2003; SILVA; DESSEN, 2006), com Qualis Al, teve um total de seis publicações, algo que é correspondente a 16,66\%. estudado.

A tabela 3 mostra a quantidade de artigos publicados ao longo do período

\begin{tabular}{|c|c|c|}
\hline Ano de publicação & N & $\%$ \\
\hline 2011 & 4 & 11,11 \\
\hline 2010 & 7 & 19,44 \\
\hline 2009 & 5 & 13,88 \\
\hline 2008 & 5 & 13,88 \\
\hline 2007 & 4 & 11,11 \\
\hline 2006 & 3 & 8,33 \\
\hline 2005 & 3 & 8,33 \\
\hline 2004 & 4 & 11,11 \\
\hline 2003 & 1 & 2,77 \\
\hline 2002 & 0 & 0 \\
\hline TOTAL & 36 & $100 \%$ \\
\hline
\end{tabular}

Tabela 3. Porcentagem de publicação anual em periódicos

Os dados da Tabela 3 apontam para um crescimento do número de publicações com famílias de pessoas com NEE nos últimos dez anos, com destaque para os anos de 2008, 2009 e 2010. Contudo, os dados não permitem atribuir uma justificativa para o aumento nesse período. Observa-se que no período referente a 2011 houve um pequeno decréscimo de publicações na temática. Na pesquisa realizada, não foi encontrada publicação no ano de 2002. 
A Tabela 4 apresenta as técnicas de coleta de dados utilizadas nas pesquisas.

\begin{tabular}{|l|c|c|}
\hline \multicolumn{1}{|c|}{ Instrumentos de coleta de dados } & $\mathrm{N}$ & $\%$ \\
\hline Análise documental, escalas e inventário & 1 & 2,77 \\
\hline Entrevista e observação & 2 & 5,55 \\
\hline Entrevista e análise documental & 3 & 8,33 \\
\hline Entrevista e questionário & 1 & 8,33 \\
\hline Entrevista, diário feito pelos pais e observação & 1 & 2,77 \\
\hline Entrevista, inventário e observação & 1 & 2,77 \\
\hline Entrevista, questionário e observação & 9 & 2,77 \\
\hline Entrevistas & 1 & 2,77 \\
\hline Entrevistas, questionários, escalas, observação e formulários & 1 & 2,77 \\
\hline Observação & 3 & 8,33 \\
\hline Questionário & 1 & 2,77 \\
\hline Questionário e escalas & 1 & 2,77 \\
\hline Questionário, inventário, teste do pensamento criativo & 1 & 2,77 \\
\hline Questionário e inventário & 1 & 2,77 \\
\hline Questionário, inventário e escalas & 1 & 11,11 \\
\hline Revisão de literatura & 1 & 2,77 \\
\hline Videogravação e observação & 1 & 2,77 \\
\hline Grupo focal & $100 \%$ \\
\hline TOTAL & 1 & 1 \\
\hline Tabel & 1 & 1 \\
\hline
\end{tabular}

Tabela 4. Porcentagem de técnicas de coleta de dados utilizadas nas pesquisas

Os dados da Tabela 4 mostram que os instrumentos mais utilizados para a coleta de dados foram: entrevistas (BOLSONI-SILVA et al., 2010; CANHO; NEME; YAMADA, 2006; CHAGAS; FLEITH, 2011; FERRAZ; ARAÚJO; CARREIRO, 2010; KRÜGER et al., 201l; PETEAN; SUGUIHURA, 2005; SILVA; MENDES, 2008; SOARES; FRANCO; CARVALHO, 2009; YAMANAKA et al., 2010) e revisão da literatura (FÁVERO; SANTOS, 2005; HENN; PICCININI; GARCIAS, 2008; KREUTZ; BOSA, 2009; LUIZ et al., 2008). A primeira com um total de nove trabalhos, correspondendo aproximadamente a $25,0 \%$, e a segunda com quatro artigos, o que equivale a $11,11 \%$. Ressalta-se que um dos trabalhos que utilizou a entrevista como técnica de coleta de dados foi uma replicação de outra pesquisa.

A Tabela 5 mostra as temáticas investigadas nos estudos empíricos. Optou-se por separar os estudos empíricos em diferentes tabelas (Tabelas 5, 6, 7, 8, 9, 10), tendo como base as temáticas trabalhadas pelos mesmos. Ressalta-se que o valor total encontrado em cada uma delas tem como ponto de referência a quantidade total de artigos investigados nesta pesquisa que totalizam 36 estudos. 
Estado da arte em revistas educacionais sobre estudos feitos com famílias de crianças com necessidades educacionais especiais no período de 2002 a 2011

\begin{tabular}{|c|c|c|}
\hline \multicolumn{3}{|l|}{ Estudos empíricos - Deficiências } \\
\hline Temáticas & $\mathrm{N}$ & $\%$ \\
\hline Síndrome de Down & 6 & 16,66 \\
\hline 1. Relação família e pessoas com síndrome de Down & 2 & 5,55 \\
\hline 2. Concepção da família sobre a educação formal dos filhos & 1 & 2,77 \\
\hline $\begin{array}{l}\text { 3. Concepção da família e professores sobre o desenvolvimento de } \\
\text { crianças com Síndrome de Down e desenvolvimento típico }\end{array}$ & 1 & 2,77 \\
\hline $\begin{array}{l}\text { 4. Relação família e filhos com Síndrome de Down e } \\
\text { desenvolvimento típico }\end{array}$ & 1 & 2,77 \\
\hline $\begin{array}{l}\text { 5. Concepção de pais e profissionais sobre a deficiência e aspectos de } \\
\text { relacionamento }\end{array}$ & 1 & 2,77 \\
\hline Surdez & 5 & 13,88 \\
\hline 1. Práticas de letramento no ambiente familiar e escolar & 1 & 2,77 \\
\hline $\begin{array}{l}\text { 2. Concepção de mães sobre surdez e a relação disso com a escolha da } \\
\text { modalidade de linguagem do filho }\end{array}$ & 1 & 2,77 \\
\hline 3. Programa de EAD de Orientação de Pais de crianças surdas & 1 & 2,77 \\
\hline 4. Vivências emocionais e mecanismos de defesa do pai & 1 & 2,77 \\
\hline 5. Concepção de pais sobre implante coclear & 1 & 2,77 \\
\hline Deficiência Intelectual & 2 & 5,55 \\
\hline $\begin{array}{l}\text { l. Impacto no desenvolvimento dos filhos diante de uma mãe com de- } \\
\text { ficiência intelectual }\end{array}$ & 1 & 2,77 \\
\hline 2. Interação entre irmãos & 1 & 2,77 \\
\hline Deficiência Múltipla & 2 & 5,55 \\
\hline 1. Inclusão escolar e social na concepção de pais e professores & 1 & 2,77 \\
\hline $\begin{array}{l}\text { 2. Análise de atos comunicativos em contextos familiares por meio de } \\
\text { registro de diário feito pela família e ralato familiar }\end{array}$ & 1 & 2,77 \\
\hline Paralisia Cerebral & 1 & 2,77 \\
\hline $\begin{array}{l}\text { 1. Característica da estrutura familiar e identificação de } \\
\text { comportamento de cuidado de irmãos de pessoas com paralisia } \\
\text { cerebral. }\end{array}$ & 1 & 2,77 \\
\hline Deficiência Física & 1 & 2,77 \\
\hline 1. Relação entre pai e filho com deficiência física & 1 & 2,77 \\
\hline TOTAL DE ESTUDOS EMPÍRICOS DEFICIÊNCIAS & 17 & 47,22 \\
\hline
\end{tabular}

Tabela 5. Principais temáticas abordadas pelos estudos empíricos deficiências e surdez

Observa-se, na Tabela 5, que as temáticas mais presentes foram síndrome de Down (CAMARGO; TOREZAN, 2004; DALLABRIDA, 2007; PEREIRA-SILVA; DESSEN, 2007; PETEAN; SUGUIHURA, 2005; SILVA; DESSEN, 2003; SILVA; DESSEN, 2006), com um total de seis temáticas, o que corresponde a $16,66 \%$. Posteriormente, a temática mais presente foi surdez (CANHO; NEME, 2006; MOTTI; PARDO, 2010; SCHEMBERG; GUARINELLO; SANTANA, 2009; SILVA; PEREIRA; 
ZANOLLI, 2007; YAMANAKA et al., 2010), sendo encontrado um total de cinco temáticas o que corresponde a 13,88\% e a deficiência intelectual (POSTALLI; MUNUERA; AIELLO, 201l; NUNES; AIELLO, 2008) e a deficiência múltipla (SILVEIRA; NENES, 2006; DELIBERATO; MANZINI; GUARDA, 2004) totalizando dois artigos o que corresponde a uma porcentagem de 5,55\%. As demais temáticas paralisia cerebral (SOARES; FRANCO; CARVALHO, 2009) e deficiência física (CHACON, 2011) totalizaram apenas um artigo o que corresponde a uma porcentagem de $2,77 \%$.

A Tabela 6 apresenta as principais temáticas abordadas pelos estudos empíricos Transtorno Global do Desenvolvimento.

\begin{tabular}{|l|c|c|}
\hline \multicolumn{3}{|c|}{ Estudos empíricos - Transtorno Global do Desenvolvimento (TDG) } \\
\hline Temáticas & $\mathrm{N}$ & $\%$ \\
\hline Autismo & 2 & 5,55 \\
\hline 1. Característica de coparentalidade entre pais & 1 & 2,77 \\
\hline $\begin{array}{l}\text { 2. Percepção de mães quanto a habilidades de interação e comu- } \\
\text { nicação do filho }\end{array}$ & 1 & 2,77 \\
\hline Distúrbio de linguagem & 1 & 2,77 \\
\hline 1. Comunicação suplementar no contexto familiar & 1 & 2,77 \\
\hline Alerações de linguagem de origem neurológica & 1 & 2,77 \\
\hline $\begin{array}{l}\text { l. Expectativa de famílias e educadores de crianças com alterções } \\
\text { de linguagem de origem neurológica }\end{array}$ & 1 & 2,77 \\
\hline Transtornos globais do desenvolvimento & 1 & 2,77 \\
\hline $\begin{array}{l}\text { l. Estresse e relação familiar em irmãos de indivíduos com } \\
\text { transtornos globais do desenvolvimento }\end{array}$ & 1 & 2,77 \\
\hline TOTAL DE ESTUDOS EMPÍRICOS TGD & 5 & 13,88 \\
\hline
\end{tabular}

Tabela 6. Principais temáticas abordadas pelos estudos empíricos Transtorno Global do Desenvolvimento

Os dados mostram que a temática mais estudada foi o autismo (SIFUENTES; BOSA, 2010; MALASPINA; LAMÔNICA, 2004) com um total de dois estudos $(5,55 \%)$. As demais temáticas distúrbios de linguagem (KRÜGER et al., 2011), alterações de linguagem de origem neurológica (TAKASE; CHUN, 2010), transtornos globais do desenvolvimento (GOMES; BOSA, 2004) possuem apenas um estudo correspondendo a 2,77\%. A Tabela 7 aborda as principais temáticas dos estudos empíricos realizados com diferentes populações.

\begin{tabular}{|l|c|c|}
\hline \multicolumn{3}{|c|}{ Estudos empíricos realizados com diferentes populações } \\
\hline Temáticas & $\mathrm{N}$ & $\%$ \\
\hline $\begin{array}{l}\text { l. Atitudes parentais diante da inclusão dos filhos com necessidades } \\
\text { educaionais especiais }\end{array}$ & 1 & 2,77 \\
\hline
\end{tabular}


Estado da arte em revistas educacionais sobre estudos feitos com famílias de crianças com necessidades educacionais especiais no período de 2002 a 2011

\begin{tabular}{|l|c|c|}
\hline $\begin{array}{l}\text { 2. Atitudes parentais diante da inclusão dos filhos com deficiência } \\
\text { auditiva e distúrbio de linguagem }\end{array}$ & 1 & 2,77 \\
\hline $\begin{array}{l}\text { 3. Parceria colaborativa entre escola e família de crianças com } \\
\text { Síndrome de Down, deficiência intelectual, deficiência visual, } \\
\text { paralisia cerebral, Síndrome de West e Síndrome de Costello }\end{array}$ & 1 & 2,77 \\
\hline $\begin{array}{l}\text { 4. Concepção de pais e professores sobre a inclusão do aluno com } \\
\text { paralisia cerebral e Síndrome de Down }\end{array}$ & 1 & 2,77 \\
\hline $\begin{array}{l}\text { 5. Comportamentos de apego entre mães e crianças com autismo, } \\
\text { Síndrome de Down e desenvolvimento típico em situação de } \\
\text { brincadeira }\end{array}$ & 1 & 2,77 \\
\hline $\begin{array}{l}\text { TOTAL DE ESTUDOS EMPÍRICOS REALIZADOS COM } \\
\text { DIFERENTES POPULAÇÕES }\end{array}$ & 5 & 13,9 \\
\hline
\end{tabular}

Tabela 7. Principais temáticas abordadas pelos estudos empíricos realizados com diferentes populações

Observa-se, na Tabela 7, que existem cinco temáticas estudadas: atitudes parentais diante da inclusão dos filhos com necessidades educacionais especiais (BARBOSA; ROSINI; PEREIRA, 2007), atitudes parentais diante da inclusão dos filhos com surdez e distúrbio de linguagem (BOLSONI-SILVA et al., 2010), parceria colaborativa entre escola e família de crianças com síndrome de Down, deficiência intelectual, deficiência visual, paralisia cerebral, síndrome de West e síndrome de Costello (SILVA; MENDES, 2008), concepção de pais e professores sobre a inclusão do aluno com paralisia cerebral e síndrome de Down (FERRAZ; ARAÚJO; CARREIRO, 2010) e comportamentos de apego entre mães e crianças com autismo, síndrome de Down e desenvolvimento típico em situação de brincadeira (SANINI et al., 2008). Sendo que cada uma delas possui um artigo. o que corresponde a porcentagem de $2,77 \%$ e uma porcentagem total de $13,88 \%$. A Tabela 8 apresenta os dados das principais temáticas abordadas pelos estudos empíricos sobre Altas Habilidades/Superdotação.

\begin{tabular}{|l|c|c|}
\hline \multicolumn{3}{|c|}{ Estudos Empíricos - Altas Habilidades/Superdotação } \\
\hline Temáticas & $\mathrm{N}$ & $\%$ \\
\hline $\begin{array}{l}\text { l. Caracterização das famílias em situação socioenconômica desfavo- } \\
\text { recida com filhos superdotados }\end{array}$ & 1 & 2,77 \\
\hline $\begin{array}{l}\text { 2. Identificação de características cognitivas, acadêmicas, afetivas e } \\
\text { sociais em adolescentes superdotados e identificação de fatores que } \\
\text { favorecem ou dificultam o desenvolvimento das habilidades }\end{array}$ & 1 & 2,77 \\
\hline TOTAL DE ESTUDOS EMPÍRICOS ALTAS HABILIDADES & 2 & 5,55 \\
\hline
\end{tabular}

Tabela 8. Principais temáticas abordadas pelos estudos empíricos Altas Habilidades/Superdotação

Os dados da Tabela 8 mostram que houve dois estudos empíricos que estudou altas habilidades (CHAGAS; FLEITH, 2009; CHAGAS; FLEITH, 2011) equivalendo a 5,55\%. A Tabela 9 refere-se as principais temáticas abordadas pelos estudos empíricos Criança de Risco e Crianças com Desenvolvimento Típico. 


\begin{tabular}{|c|c|c|}
\hline \multicolumn{3}{|l|}{ Estudos Empíricos de outras categorias } \\
\hline Temáticas - Crianças de risco & $\mathrm{N}$ & $\%$ \\
\hline $\begin{array}{l}\text { 1. Impacto de uma intervenção com a família e os seus reflexos no } \\
\text { desenvolvimento da criança }\end{array}$ & 1 & 2,77 \\
\hline Temáticas - Criança com desenvolvimento típico & $\mathrm{N}$ & $\%$ \\
\hline $\begin{array}{l}\text { 1. Envolvimento dos pais em intervenção precoce por meio de aplica- } \\
\text { ção de um questionário }\end{array}$ & 1 & 2,77 \\
\hline Transtorno de déficit de atenção e hiperatividade & 1 & 2,77 \\
\hline $\begin{array}{l}\text { 1. Estresse parental em mães de crianças com transtorno de déficit de } \\
\text { atenção e hiperatividade e desenvolvimento típico }\end{array}$ & 1 & 2,77 \\
\hline TOTAL DE ESTUDOS EMPÍRICOS DE OUTRAS CATEGORIAS & 3 & 8,33 \\
\hline
\end{tabular}

Tabela 9. Principais temáticas abordadas pelos estudos empíricos de outras categorias

A Tabela 9 mostra que existe apenas um estudo empírico realizado com crianças de risco (CIA; WILLIAMS; AIELLO, 2005), um com criança com desenvolvimento típico (GRAÇA et al., 2010) e um com criança com transtorno de déficit de atenção e hiperatividade (BELLÉ et al., 2009), correspondendo a 2,77\% cada um deles. A Tabela 10 apresenta as principais temáticas abordadas pelos estudos de revisão de literatura.

\begin{tabular}{|l|c|c|}
\hline \multicolumn{3}{|c|}{ Revisão da Literatura } \\
\hline Variáveis & $\mathrm{N}$ & $\%$ \\
\hline Síndrome de Down & 2 & 5,55 \\
\hline 1. Inclusão escolar & 1 & 2,77 \\
\hline 2. Impacto da síndrome de Down na família & 1 & 2,77 \\
\hline Deficiência Visual & 1 & 2,77 \\
\hline 1. Intervenção precoce e relação família-criança & 1 & 2,77 \\
\hline Autismo & 1 & 2,77 \\
\hline 1. Impacto psicossocial em famílias & 1 & 2,77 \\
\hline TOTAL DE ESTUDOS DE REVISÃO DE LITERATURA & 4 & 11,11 \\
\hline
\end{tabular}

Tabela 10. Principais temáticas abordadas pelos estudos de revisão de literatura

Os dados permitem observar em estudos que utilizaram como técnica de coleta de dados a revisão da literatura, a temática mais presente é a síndrome de Down, que totaliza dois artigos, sendo abordados dois assuntos diferentes, entre eles inclusão escolar (LUIZ et al., 2008) e impacto da síndrome de Down na família (HENN; PICCININI; GARCIAS, 2008). Nas demais temáticas, deficiência visual (KREUTZ; BOSA, 2009) e autismo (FAVERO; SANTOS, 2005) foram encontrados apenas um estudo em cada uma, o que corresponde a uma porcentagem de $2,77 \%$. 


\section{Discussão}

Observou-se que muitos estudos analisados procuraram investigar a família de crianças com NEE sob uma concepção sistêmica, demonstrando interesse, principalmente, pelas relações e interações estabelecidas nesse contexto e inserindo como participantes, além da mãe, (BELLÉ et al., 2009; MALASPINA; LAMÔNICA, 2004; SANINI et al., 2008; SILVA; PEREIRA; ZANOLLI, 2007;), o pai (CANHO; LEME, 2006; CHACON, 2011) e os irmãos das pessoas com NEE (GOMES; BOSA, 2004; NUNES; AIELLO, 2008; SOARES; FRANCO; CARVALHO, 2009). Isso vai ao encontro das pesquisas de Dessen (1997) e Dessen e Braz (2005) que constataram um aumento nos últimos anos de pesquisas que investigaram as diversas relações estabelecidas entre os atores familiares.

Outro destaque está nas pesquisas que investigaram o impacto de uma criança com NEE na família ou a relação familiar estabelecida com essa criança ou, ainda, a caracterização da família dessas crianças (CHAGAS; FLEITH, 2009; CIA; WILLIAMS; AIELLO, 2005; FÁVERO; SANTOS, 2005; HENN; PICCININI; GARCIAS, 2008; PETEAN; SUGUIHURA, 2005; SILVA; DESSEN, 2003; SILVA; DESSEN, 2006;). Além disso, as pesquisas apontaram para uma preocupação sobre a concepção da família em relação à educação do filho e também para as concepções de pais e professores sobre o processo de inclusão das crianças (BARBOSA; ROSINI; PEREIRA, 2007; BOLSONI-SILVA et al., 2010; CAMARGO; TOREZAN, 2004; DALLABRIDA, 2007; FERRAZ; ARAÚJO; CARREIRO, 2010; LUIZ et al., 2008; PEREIRA-SILVA; DESSEN, 2007; SCHEMBERG; GUARINELLO; SANTANA; 2009; SILVA; MENDES, 2008; SILVEIRA; NENES, 2006; TAKASE; CHUN, 2010). Este dado é relevante, uma vez que demonstra a preocupação com o processo de inclusão de crianças com NEE e a necessidade de existir parceria entre a escola e a família. Sobre isso, Paniagua e Palacios (2008) apontam para o estabelecimento de uma relação de colaboração entre a família e a escola:

Em vez de nos propormos a ensinar aos pais, temos mais é que aprender uns com os outros. Assim, as famílias nos ensinam como são seus filhos ou filhas em casa, quais são as propostas educativas no seu meio, entre outros itens. Quando as famílias se sentem realmente ouvidas e respeitadas, também se mostram mais predispostas a ouvir e a aprender. (p. 217)

Assim, em se tratando das relações estabelecidas entre família e profissionais, é importante que os programas de atendimento auxiliem no empoderamento das famílias, a fim de torná-las promotoras do desenvolvimento da criança (DESSEN; CERQUEIRA-SILVA, 2008; WILLIAMS; AIELLO, 2004).

No que diz respeito às técnicas de coleta de dados, observou-se que a entrevista foi o procedimento mais utilizado pelos estudos. Este dado também é interessante, pois sugere uma preocupação em ouvir a família de crianças com NEE, assim como os profissionais que as atendem, demonstrando interesse e cuidado em criar uma parceria entre a família e o atendimento, de modo que ambos contribuam para a educação da criança. 
Quanto às temáticas abordadas pelos artigos analisados, elas foram bastante diversificadas, o que indicia um crescimento nas pesquisas de famílias de crianças com NEE. Destaca-se o aumento no número de pesquisas empíricas, se comparadas às pesquisas de revisão de literatura. Tal aspecto é relevante, pois a pesquisa de Goitein e Cia (2011) identificou um maior número de pesquisas de revisão da literatura em periódicos indexados na área da Psicologia, entre os anos de 1999 a 2008. Apesar de o período e a área de indexação do referido estudo não coincidirem com essa pesquisa este dado é relevante, haja vista que revela um crescimento de estudos empíricos que abordam famílias de pessoas com NEE e um interesse da área educacional em abordar esse assunto.

Diante do exposto, percebe-se uma modificação nas pesquisas que tratam de famílias de crianças com NEE. Há um predomínio de interesse em pesquisas empíricas que investigam diferentes atores da família. Nestas pesquisas não mais somente a mãe figura entre os principais interesses, mas o pai e os irmãos da criança com NEE, apontando a importância em ouví-los, para, assim, empoderá-los no sentido de serem agentes participativos do processo educativo da criança com NEE.

\section{Considerações finais}

Essa revisão da literatura permitiu observar que houve um crescimento de pesquisas empíricas com famílias de crianças com NEE ao longo da última década, demonstrando uma preocupação em conhecer a realidade das famílias, o conhecimento que elas têm sobre as necessidades do filho(a), além de investigar as concepções que família e profissionais que cuidam de crianças com necessidades especiais possuem acerca deste universo de convivência. Isso ressalta a importância da parceria entre profissionais e família, a fim de que ambos possam contribuir para o processo educativo da criança. Entretanto, para que isso possa ser investigado de forma mais pontual, são necessários mais estudos de caráter longitudinal, conforme sugerido por Dessen e Braz (2005) e Goitein e Cia (2011), a fim de que possam investigar as relações da família de pessoas com NEE e também a relação entre ela e os profissionais que atendem seu filho(a).

Por fim, a amostra dessa pesquisa consistiu em analisar os estudos brasileiros indexados na área da educação e avaliados, no triênio 2010-2012, como Al e A2, mas sugere-se que outros estudos sejam feitos com uma amostragem maior, abrangendo pesquisas internacionais e as indexadas em outras áreas do conhecimento científico.

\section{Referências}

BARbOSA, A. J. G.; ROSINI, D. C.; PEREIRA, A. A. Atitudes parentais em relação à educação inclusiva. Revista Brasileira de Educação Especial, Marília, v. 13, n. 3, p. 447-458, 2007.

BRASIL. Ministério da Educação. Secretaria de Educação Continuada, Alfabetização, Diversidade e Inclusão (SECADI). Política Nacional de Educação Especial na Perspectiva da Educação Inclusiva. Brasília, 2007 . 
Estado da arte em revistas educacionais sobre estudos feitos com famílias de crianças com necessidades educacionais especiais no período de 2002 a 2011

BEE, H. A criança em desenvolvimento. Tradução de Cristina Monteiro. 12 ed. Porto Alegre: Artmed, 2011.

BELLÉ, A.H.; ANDREAZZA, A.C.; RUSCHEL, J.; BOSA, C.A. Estresse e adaptação psicossocial em mães de crianças com transtorno de déficit de atenção/hiperatividade. Psicologia: Reflexão e Crítica, Porto Alegre, v. 22, n. 3, p. 317-325, 2009.

BOLSONI-SILVA, A.T.; RODRIGUES, O.M.P.R.; ABRAMIDES, D.V.M.; SOUZA, L.S.; LOUREIRO, S.R. Práticas educativas parentais de crianças com deficiência auditiva e de linguagem. Revista Brasileira de Educação Especial, Marília, v. 16, n. 2, p. 265-282, 2010.

CAMARGO, E. A. A.; TOREZAN, A. M. Interlocução entre pais e profissionais da área de educação especial e suas concepções sobre a deficiência mental. Revista Brasileira de Educação Especial, Marília, v. 10, n. 3, p. 337-354, 2004.

CANHO, P. G. M.; NEME, C. M. B.; YAMADA, M. O. A vivência do pai no processo de reabilitação da criança com deficiência auditiva. Estudos de Psicologia, Campinas, v. 23, n. 3, p. 261-269, 2006.

CHACON, M. C. M. Aspectos relacionais, familiares e sociais da relação pai-filho com deficiência física. Revista Brasileira de Educação Especial, Marília, v. 17, n. 3, p. 441-458, 2011.

CHAGAS, J. F.; FLEITH, D.S. Estudo comparativo sobre superdotação com famílias em situação socioeconômica desfavorecida. Revista Brasileira de Educação Especial, Marília, v. 15, n. 1, p. 155-170, 2009.

CHAGAS, J. F.; FLEITH, D.S. Perfil de adolescentes talentosos e estratégias para o seu desenvolvimento. Psicologia: Teoria e Pesquisa, Brasília, v. 27, n. 4, p. 385-392, 2011.

CIA, F.; WILLIAMS, L. C. A.; AIELLO, A. L. R. Intervenção focada na família: Um estudo de caso com mãe adolescente e criança de risco. Revista Brasileira de Educação Especial, Marília, v. 1l, n. 1, p. 49-66, 2005.

DALLABRIDA, A. M. As famílias e a classe especial em um colégio de elite. Revista Brasileira de Educação Especial, Marília, v. 13, n. 3, p. 459-478, 2007.

DELIBERATO, D.; MANZINI, E. D.; GUARDA, N. S. Implementação de recursos suplementares de comunicação: participação da família na descrição de comportamentos comunicativos dos filhos. Revista Brasileira de Educação Especial, Marília, v. 10, n. 2, p. 217-240, 2004.

DESSEN, M.A. Desenvolvimento familiar: transição de um sistema triádico para poliádico. Temas em Psicologia, Ribeirão Preto, v. 3, p. 5l-61. 1997.

DESSEN, M. A.; LEWIS, C. Como estudar a família e o "pai". Paidéia. Ribeirão Preto, v. 8, n. 14-15, p. 105121, 1998.

DESSEN, M.A.; CERQUEIRA-SILVA, S. Famílias e crianças com deficiência: em busca de estratégias para promoção do desenvolvimento familiar. In: BOLSANELLO, M.A. (Org.). Atenção e estimulação precoce. Curitiba: I Simpósio Nacional de Atenção e Estimulação Precoce, p. 39-58, 2008.

DESSEN, M. A.; BRAZ, M. P. A família e suas inter-relações com o desenvolvimento humano. In: DESSEN, M. A.; COSTA-JUNIOR, A. L. A ciência do desenvolvimento humano: tendências atuais e perspectivas futuras. Porto Alegre: Artmed, 2005. p. 113-131.

FÁVERO, M. A. B.; SANTOS, M. A. Autismo infantil e estresse familiar: uma revisão sistemática da literatura. Psicologia: Reflexão e Crítica, Porto Alegre, v. 18, n. 3, p. 358-369, 2005.

FERRAZ, C.R.A.; ARAÚJO, M.V.; CARREIRO, L.R.R. Inclusão de crianças com síndrome de Down e paralisia cerebral no ensino fundamental I: comparação dos relatos de mães e professores. Revista Brasileira de Educação Especial, Marília, v. 16, n. 3, p. 397-414, 2010.

Revista Educação Especial | v. 27 | n. 48 | p. 201-218 | jan./abr. 2014

Santa Maria

Disponível em: <http://www.ufsm.br/revistaeducacaoespecial> 
GEMA, P. As famílias de crianças com necessidades educativas especiais. In: COLL, C.; MARCHESI, A.; PALACIOS, J. (Orgs.). Desenvolvimento psicológico e educação: transtorno de desenvolvimento e necessidades educativas especiais. Porto Alegre: Artmed, v. 3, p. 331-347, 1995.

GOITEN, P. C.; CIA, F. Interações familiares de crianças com necessidades educacionais especiais: revisão de literatura nacional. Revista Semestral da Associação Brasileira de Psicologia Escolar e Educacional, São Paulo, v. 15, n. 1, p. 43-51, 2011.

GOMES, V. F.; BOSA, C. Estresse e relações familiares na perspectiva de irmãos de indivíduos com TGD. Estudos de Psicologia, Natal, v. 9, n. 3, p. 553-561, 2004.

GRAÇA, P.R.M.; TEIXEIRA, M.L.S.C.; LOPES, S.C.G.; SERRANO, A.M.S.P.H.; CAMPOS, A.R.S. O momento da avaliação na intervenção precoce: o envolvimento da família estudo das qualidades psicométricas do ASQ 2 dos 30 aos 60 meses. Revista Brasileira de Educação Especial, Marília, v. 16, n. 2, p. 177-196, 2010.

HENN, C. G.; PICCININI, C. A.; GARCIAS, G. L. A família no contexto da síndrome de Down: revisando a literatura. Psicologia em Estudo, Maringá, v. 13, n. 3, p. 485-493, 2008.

JUNIOR, G. A. F.; MESSA, A. A. Pais, filhos e deficiência: estudos sobre as relações familiares. Psicologia Ciência e Profissão, Brasília, v. 27, n. 2, p. 236-245. 2007.

KREUTZ, C. M.; BOSA, C. A. Intervenção precoce na comunicação pais-bebê com deficiência visual. Estudos em Psicologia. Campinas, v. 26, n. 4, p. 537-544, 2009.

KRÜGER, S.; BERBERIAN, A.P.; GUARINELLO, A.C.; CARNEVALE, L.B. Comunicação suplementar e/ ou alternativa: fatores favoráveis e desfavoráveis ao uso no contexto familiar. Revista Brasileira de Educação Especial, Marília, v. 17, n. 2, p. 209-224, 2011.

LUIZ; F.M.R.; BORTOLI, P.S.; FLORIA-SANTOS, M.; NASCIMENTO, L.C. Inclusão da criança com síndrome de Down na rede regular de ensino: Desafios e possibilidades. Revista Brasileira de Educação Especial, Marília, v. 14, n. 3, p. 497-508, 2008.

MALASPINA, E. A.; LAMÔNICA, D. A. C. Habilidades interativas e comunicativas de crianças autistas: ponto de vista materno. Revista Brasileira de Educação Especial, Marília, v. 10, n. 2, p. 221-234, 2004.

MOTTI, T.F.G.; PARDO, M.B.L. Intervenção com pais de crianças deficientes auditivas: elaboração e avaliação de um programa de orientação não presencial. Revista Brasileira de Educação Especial, Marília, v. 16, n. 3, p. 447-462, 2010

NUNES, C. C., AIELLO, A. L. R. Interação entre irmãos: deficiência mental, idade e apoio social da família. Psicologia: Reflexão e Crítica, Porto Alegre, v. 21, n. 1, p. 42-50. 2008.

PANIAGUA, G.; PALACIOS, J. Relações com famílias. In: ___ (Orgs.). Educação Infantil: resposta educativa à diversidade. Tradução de Fátima Murad. Porto Alegre: Artmed, 2007, p. 211-234.

PEREIRA-SILVA, N. L.; DESSEN, M. A. Crianças com e sem síndrome de Down: Valores e crenças de pais e professores. Revista Brasileira de Educação Especial, Marília, v. 13, n. 3, p. 429-446, 2007.

PETEAN, E.B.L.; SUGUIHURA, A.L.M. Ter um irmão especial: convivendo com a síndrome de Down. Revista Brasileira de Educação Especial, Marília, v. 1l, n. 3, p. 445-460, 2005.

POSTALLI, L. M. M.; MANUERA, R. F.; AIELLO, A. L. R. Caracterização de família de mãe com deficiência intelectual e os efeitos no desenvolvimento dos filhos. Revista Brasileira de Educação Especial, Marília, v. 17, n. 1, p. 37-52, 2011. 
Estado da arte em revistas educacionais sobre estudos feitos com famílias de crianças com necessidades educacionais especiais no período de 2002 a 2011

SANINI, C.; FERREIRA, G. D.; SOUZA, T. S.; BOSA, C. A. Comportamentos indicativos de apego em crianças com autismo. Psicologia: Reflexão e Crítica, Porto Alegre, v. 21, n. 1, p. 60-65. 2008.

SCHEMBERG, S.; GUARINELLO, A. C.; SANTANA, A.P.O. As práticas de letramento na escola e na família no contexto da surdez: reflexões a partir do discurso dos pais e professores. Revista Brasileira de Educação Especial, Marília, v. 15, n. 2, p. 251-268, 2009.

SIFUENTES, M.; BOSA, C. A. Criando pré-escolares com autismo: Características e desafios da coparentalidade. Psicologia em Estudo, Maringá, v. 15, n. 3, p. 477-485, 2010.

SILVA, N. L. P.; DESSEN, M. A. Deficiência mental e família: implicações para o desenvolvimento da criança. Psicologia: Teoria e Pesquisa, Brasília, v. 17 n. 2, p. 133-141, 2001.

SILVA, N. L. P.; DESSEN, M. A. Crianças com síndrome de Down e suas interações familiares. Psicologia: Reflexão e Crítica, Porto Alegre, v. 16, n. 3, p. 503-514, 2003.

SILVA, N. L. P.; DESSEN, M. A. Padrões de interação genitores-crianças com e sem síndrome de Down. Psicologia: Reflexão e Crítica, Porto Alegre, v. 19, n. 2, p. 283-291. 2006.

SILVA, A. M.; MENDES, E. G. Família de crianças com deficiência e profissionais:componentes da parceria colaborativa na escola. Revista Brasileira de Educação Especial, Marília, v. 14, n. 2, p. 217-234, 2008.

SILVA, A. B. P.; PEREIRA, M. C. C.; ZANOLLI, M. L. Mães ouvintes com filhos surdos: concepção de surdez e escolha da modalidade de linguagem. Psicologia: Teoria e Pesquisa, Brasília, v. 23, n. 3, p. 279-286, 2007.

SILVEIRA, F. F.; NEVES, M. M. B. J. Inclusão escolar de crianças com deficiência múltipla: concepção de pais e professores. Psicologia: Teoria e Pesquisa, Brasília, v. 22, n. 1, p. 079-088, 2006.

SOARES, M. P. G.; FRANCO, A. L. S.; CARVALHO, A. M. A. Crianças que cuidam de irmãos com necessidades especiais. Psicologia: Teoria e Pesquisa, Brasília, v. 25 n. 1, p. 45-54, 2009.

TAKASE, E.M.; CHUN, R.Y.S. Comunicação e inclusão de crianças com alterações de linguagem de origem neurológica na perspectiva de pais e educadores. Revista Brasileira de Educação Especial, Marília, v. 16, n. 2, p. $251-264,2010$.

WILliAMS, L. C. A.; AIELLO, A. L. R.; Empoderamento de famílias: o que vem a ser e como medir. In: MENDES, E. G.; ALMEIDA, M. A.; WILLIAMS, L. C. A. (Orgs.). Temas em educação especial: avanços recentes. São Carlos: EDUFSCar, 2004, p. 197-202.

YAMANAKA, D.A.R.; SILVA, R.B.P.; ZANOLLI, M.L.; SILVA, A.B.P. Implante coclear em crianças: A visão dos pais. Psicologia: Teoria e Pesquisa, Brasília, v. 26 n. 3, p. 465-473, 2010.

\section{Correpondência}

Giovana Mendes Ferroni - Universidade Federal de São Carlos, Centro de Educação e Ciências Humanas, Departamento de Educação Especial. Rodovia Washington Luiz, Km 235, CEP: 13565-305, São Carlos, São Paulo, Brasil.

E-mail: giovanaferroni@yahoo.com.br-fabianacia@ufscar.br

Recebido em 26 de março de 2013

Aprovado em 25 de abril de 2013 
